\title{
Reincidentes en el cuidado, pero sin derecho a la prevención: un análisis de la oferta de la profilaxis posexposición sexual al VIH en Porto Alegre, Brasil
}

\author{
'Repeat offenders' in care, but with no right to \\ prevention: An analysis of the availability of post- \\ exposure prophylaxis for HIV in Porto Alegre, Brazil
}

Bruno Kauss ${ }^{1}$, Andréa Fachel Leal2 ${ }^{2}$, Alexandre Grangeiro ${ }^{3}$, Marcia Thereza Couto ${ }^{4}$

${ }^{1}$ Autor de correspondencia. Magíster en Políticas Públicas. Investigador, Faculdade de Medicina, Universidade de São Paulo, São Paulo, Brasil. $\square$ (iD) ${ }^{2}$ Doctora en Antropología Social. Profesora Asociada IV, Instituto de Filosofía y Ciencias Humanas, Universidade Federal del Rio Grande del Sul, Porto Alegre, Brasil. $\square$ (iD ${ }^{3}$ Licenciado en Ciencias Sociales. Investigador, Faculdade de Medicina, Universidade de São Paulo, São Paulo, Brasil. $\square$ (iD)

${ }^{4}$ Doctora en Sociología. Profesora Asociada, Departamento de Medicina Preventiva, Faculdade de Medicina, Universidade de São Paulo, São Paulo, Brasil. $\triangle$ (iD

RESUMEN Buscamos identificar los desafíos para la implementación de la profilaxis posexposición sexual al VIH, a partir de analizar el accionar de las y los profesionales de la salud en un servicio de salud pública en Porto Alegre, Rio Grande do Sul, Brasil. Desde un enfoque cualitativo, con técnicas de observación etnográfica y entrevistas en profundidad, se encontró que los factores contextuales, organizacionales e individuales eran desafíos para implementar la profilaxis posexposición sexual al VIH. Las barreras para su implementación incluyeron el contexto histórico de la estructuración y la actuación del servicio, la falta de capacitación y/o educación continua en salud, y las concepciones de las y los profesionales de la salud (ideas sobre la estrategia en sí, y sobre las personas que buscan PEP). Se concluye que existe la necesidad de mayor atención al universo de servicios especializados en ITS/VIH/sida y a las y los profesionales que componen estos servicios, a fin de garantizar una mayor efectividad en el acceso a la estrategia a nivel local.

PALABRAS CLAVES Profilaxis Posexposición; Infecciones de Transmisión Sexual; VIH; Brasil.

ABSTRACT This study seeks to identify challenges in the implementation of post-exposure prophylaxis for HIV, based on an analysis of actions taken by healthcare professionals in the state-run health sector in Porto Alegre, Brazil. Based on a qualitative approach that included ethnographic observations and in-depth interviews, we found that contextual, institutional, and individual factors represented challenges to the implementation of post-exposure prophylaxis for HIV. Barriers to implementation included the historical context structuring healthcare services and practices, the lack of training and/or continued education in health, and certain attitudes on the part of healthcare professionals (ideas regarding both the strategy itself as well as the individuals that seek PEP). We conclude that there is a need for greater attention to specialized services for STI/HIV/AIDS as well as the professionals that provide these services, in order to guarantee greater effective access to this strategy at the local level.

KEY WORDS Post-Exposure Prophylaxis; Sexually Transmitted Diseases; HIV; Brazil. 


\section{INTRODUCCIÓN}

La profilaxis posexposición sexual consensuada al VIH -PEP sexual, o simplemente PEP- es una estrategia de prevención del VIH que implica el uso de medicamentos antirretrovirales durante 28 días consecutivos, por parte de aquellas personas que no viven con VIH, y que hayan tenido, en las últimas 72 horas, una relación sexual consensuada y desprotegida, por falla o no uso de algún medio de prevención del $\mathrm{VIH}^{(1)}$. La PEP integra la denominada "prevención combinada", una política internacional de enfrentamiento a la epidemia de VIH/sida, avalada por la Organización Mundial de la Salud (OMS) y el Programa Conjunto de las $\mathrm{Na-}$ ciones Unidas sobre el VIH/sida (ONUSIDA) desde $2010^{(2)}$. En líneas generales, la prevención combinada constituye un cambio de paradigma en la prevención del VIH, al reunir todos los métodos preventivos con efectividad verificada. Cobró mayor protagonismo con la incorporación de métodos preventivos que utilizan el uso de los antirretrovirales como forma de evitar la transmisión (en el caso de personas infectas por el virus) y la adquisición (en el caso de personas no infectadas) del VIH. Este nuevo paradigma permitió avanzar en estrategias preventivas del VIH y demás infecciones de transmisión sexual (ITS), más allá de la promoción del uso del preservativo, que marcó las primeras décadas de enfrentamiento de la epidemia ${ }^{(1,3,4)}$. Al mismo tiempo, les permitió a los individuos elegir el método que más se adecue a su contexto $\mathrm{y}$ necesidades sexuales. Las críticas a la prevención combinada se centran en la poca relevancia dada a las intervenciones estructurales y en la supremacía de los métodos de prevención y/o tratamiento con antirretrovirales, lo que ha sido interpretado como una biomedicalización de la prevención ${ }^{(5)}$.

La prevención combinada también integra la Meta 90-90-90, por medio de la cual los Estados miembros de la ONU se comprometieron a alcanzar, para el año 2020, un $90 \%$ de personas con VIH diagnosticadas, un $90 \%$ con tratamiento antirretroviral y, de estas, un $90 \%$ con la carga viral indetectable, lo que impide la transmisibilidad del VIH. Se estima que si la Meta 90-90-90 se alcanzara en 2020, en 2030 se decretaría el fin de la epidemia. Mientras la ciencia y los discursos biomédicos afirman la proximidad del "fin del sida", se destaca que la complejidad de la epidemia y las barreras de acceso a la salud desafían el cumplimiento de ese objetivo, presentando el "fin del sida" más como retórica que como una realidad a ser concretada ${ }^{(6)}$.

En sintonía con las recomendaciones internacionales, en Brasil se implementaron nuevas estrategias, entre las cuales se incluye la profilaxis pre y posexposición, que genera desafíos para el Sistema Único de Salud (SUS), dado que la estructura existente en los servicios no siempre se adecua a lo que dictan los protocolos clínicos ${ }^{(7,8)}$. En Brasil, la recomendación del uso profiláctico de los medicamentos antirretrovirales, en una situación de potencial exposición al VIH, se adoptó en la década de 1990 solo para los casos de accidentes ocupacionales de las y los profesionales de la salud con material biológico (exceptuando los casos de transmisión vertical). En la misma década, mujeres y adolescentes víctimas de violencia sexual fueron incluidos en la recomendación. En 2010, la profilaxis se extendió a las situaciones de exposición al VIH en relaciones sexuales consensuadas. Solo en 2015 , las recomendaciones de la profilaxis se unificaron en un único documento ${ }^{(1)}$.

La demanda de uso de la PEP aumentó, principalmente, entre personas que se identifican como gays y hombres que tienen sexo con otros hombres ${ }^{(9,10,11,12)}$. Uno de los temores señalados con relación a la PEP es la posibilidad de provocar el aumento de prácticas sexuales de riesgo al VIH y el uso repetido de la estrategia( ${ }^{(13)}$. Con base en la bibliografía, aún no es posible afirmar que la profilaxis posexposición genere la desinhibición del uso de métodos tradicionales, como los preservativos $^{(11,14)}$. El no uso de los métodos tradicionales ha sido señalado por la bibliografía internacional $^{(15)}$ y nacional ${ }^{(16)}$ como un fenómeno social, independientemente del conocimiento de los sujetos sobre las formas existentes de prevención del VIH. Para analizar el posible aumento en la búsqueda de estrategias de prevención PEP y PrEP, se necesitan todavía más estudios sobre la relación de este incremento con la desinhibición del uso de métodos tradicionales.

En este sentido, una investigación sobre la relación entre el uso de PEP y comportamientos sexuales de riesgo de infección por $\mathrm{VIH}$, en una cohorte de hombres homosexuales realizada en Sydney, Australia, concluyó que la adopción de la 
estrategia no genera el aumento de prácticas de infección por $\mathrm{VIH}^{(17)}$. Otro estudio, realizado en Rio de Janeiro, Brasil, también concluyó que no había una relación entre el aumento de prácticas sexuales de riesgo y la adopción de la prevención antirretroviral posexposición. Por lo tanto, no es posible afirmar que la PEP produce un aumento de la vulnerabilidad al $\mathrm{VIH}^{(18)}$.

En el actual contexto de ampliación de nuevas estrategias de prevención basadas en antirretrovirales, se torna importante investigar los desafíos de la oferta de PEP en el ámbito local. Analizar la implementación de una política pública, en el ámbito local, en aquellos servicios que prestan asistencia directa a los beneficiarios de una política es un estudio que involucra al nivel de la "burocracia callejera" o "primera línea"(19) de las políticas públicas. En la primera línea, el contacto de las y los profesionales con la población se ejerce constante y directamente en un proceso de traducción del instrumental normativo de las políticas públicas a la realidad local. Los estudios de implementación de políticas públicas consideran que la primera línea conjuga el conjunto de percepciones, acciones e interacciones de los trabajadores en las instituciones, como también el contexto en el cual están insertos ${ }^{(19,20,21,22)}$.

Nuestro objetivo es identificar los desafíos de la implementación de la profilaxis posexposición al VIH (PEP), a partir de analizar el accionar de las y los profesionales de la salud, en un servicio público especializado en ITS/VIH/sida en Porto Alegre, Rio Grande do Sul, Brasil. El enfoque apunta a identificar cómo estos profesionales usan sus atribuciones para interpretar planos, ejecutar tareas y alcanzar los objetivos propuestos por la política pública. Desde esta perspectiva, la pregunta de investigación se propone analizar ¿cuáles son los desafíos en el ámbito de la implementación de la PEP y cómo pueden influir en el acceso de los sujetos a la estrategia?

Aún son pocos los estudios cualitativos con enfoque en la implementación de un programa y ejecución de tareas por parte de las y los profesionales en el ámbito de los servicios de salud en Brasil. Los datos presentados integran la investigación titulada $A$ efetividade da profilaxia da transmissão do HIV pós-exposição sexual consensual (PEP), do uso combinado dos métodos preventivos contra a infecção pelo HIV e da profilaxia pré-exposição sexual (PrEP), em serviços públicos brasileiros o, simplemente, Estudio Combina, financiado por el Ministerio de Salud de Brasil (027941/2012) y por el Conselho Nacional de Desenvolvimento Científico e Tecnológico (14/2014), que contempla dos componentes epidemiológicos y uno cualitativo. El análisis realizado en este artículo es un recorte del componente cualitativo.

\section{METODOLOGÍA}

Se trata de un recorte de un estudio mayor, con abordaje cualitativo, que se enmarca en el proyecto Combina, estudio multicéntrico desarrollado en cinco ciudades brasileñas ${ }^{(23,24)}$. El diseño de "estudio de caso" para el municipio de Porto Alegre se debe al hecho de que, entre las restantes ciudades, esta presentó características propicias para la realización de un estudio de este tipo. Por un lado, contaba con una prevalencia e incidencia de VIH y una mortalidad por sida ${ }^{(25)}$ de tal magnitud, que se la denomina "capital nacional del sida" (26) $y$, por otro, las condiciones de estructura y organización del Servicio de Asistencia Especializada en ITS/VIH/sida, compuesto por dos equipos: uno al frente del centro de testeo y consejería $\mathrm{y}$, otro, del servicio ambulatorio especializado. Sumado a esto, la participación del servicio en el proyecto multicéntrico fue fruto del acuerdo entre la coordinación del proyecto Combina y la Secretaría Municipal de Salud de Porto Alegre. La Secretaría propuso este servicio por su alto volumen de atención, su tiempo de existencia (más de una década) y por haber implementado un protocolo con oferta de PEP que se encontraba vigente. La investigación de campo se realizó durante ocho meses, entre 2015 y 2016.

En la observación, de cuño etnográfico, se implementó una guía semiestructurada para la producción de datos empíricos. Siguiendo la guía, se observaron los espacios del servicio, los ambientes de recepción de personas que buscaban profilaxis, la sala de espera, los consultorios en los que se atendían a las personas y los espacios de interacción de las y los profesionales (sala de reuniones y la cocina). Entre las situaciones, se observaron la recepción, las consultas, las reuniones y los momentos de intervalos de las y los profesionales. En 
estos escenarios y situaciones, la observación se centró en las relaciones de las y los profesionales entre sí y con las personas, buscando caracterizar los procesos de toma de decisiones de las y los profesionales con relación a la PEP.

Las guías de observación tuvieron la finalidad de conocer la organización de la atención de la PEP en el servicio, y aprehender la percepción y la práctica cotidiana de las y los profesionales de la salud frente a la PEP. Además de la anuencia de la participación del servicio en la investigación más amplia realizada por la Secretaría Municipal de Salud, la entrada al campo se acordó con la coordinación del servicio y el investigador responsable del estudio y de la observación etnográfica, que no es un profesional de la salud. De cierto modo, esto aseguró un lugar de extrañamiento doble: de él, en la condición de observador, y del equipo que tenía ahora con su presencia alguien que "no era de la salud". La PEP, aún en proceso de implementación, representaba una novedad tanto para el equipo como para el investigador. El extrañamiento y la novedad fueron los puntos de partida para la construcción de una relación de confianza, y de complicidad, incluso entre el investigador y el equipo. La inserción en el servicio de asistencia especializada en ITS/ $\mathrm{VIH} /$ sida le permitió al investigador observar el día a día de las y los profesionales, la circulación de las personas en búsqueda de la profilaxis, las reuniones de equipo, las interacciones entre las unidades observadas, entre otras acciones.

La producción de los datos empíricos se basó en la técnica de entrevista en profundidad, debido a su potencialidad de captar, a partir de una guía predefinida y flexible, los valores y los sentidos que los sujetos atribuyen a sus experiencias personales y profesionales ${ }^{(27)}$. En las entrevistas, se exploraron temas como el conocimiento acerca de la PEP, se incluyeron fuentes de información y educación continua, la visión acerca de las personas y las situaciones clínicas, y cómo se daban las interacciones y orientaciones por parte de las y los profesionales con relación a estrategias para la reducción del riesgo. En las entrevistas, se convocó a todo el plantel profesional y a la gerencia del servicio. Entre los invitados, tres médicos y un técnico de enfermería se rehusaron a participar de las entrevistas. Con excepción del técnico en enfermería, los demás profesionales no estaban involucrados de forma directa, ni indirecta, con la PEP.
Los entrevistados que aceptaron participar fueron aquellos que tenían mayor contacto y compromiso con la implementación de la estrategia en el servicio. En todo momento se preservó su identidad, y sus nombres fueron remplazados para asegurar la confidencialidad. La opción de no identificar la profesión, el sexo, el género, la edad o cualquier otro atributo de las y los entrevistados fue motivada por la posibilidad de que los profesionales fueran identificados, ya que el municipio de Porto Alegre tiene pocos servicios especializados en VIH. Por lo tanto, en algunos momentos del texto se optó por el masculino genérico.

En total, se realizaron quince entrevistas a profesionales de la salud, entre ellos: un médico, dos enfermeros, un asistente social, tres psicólogos, un farmacéutico, tres técnicos de enfermería, un auxiliar de enfermería, un auxiliar administrativo y dos residentes en salud colectiva; todos, excepto por los residentes, estaban en el servicio de salud desde 2010, cuando se incorporó la PEP. Uno de los profesionales actuaba como gerente del servicio. Las entrevistas se grabaron y transcribieron, y las observaciones se registraron en el diario de campo. Se solicitó a todos la firma del consentimiento libre e informado. El protocolo de investigación fue aprobado por los Comités de Ética de la Facultad de Medicina de la Universidad de San Pablo (CAAE 34145314.5.1001.0065) y de la Secretaría Municipal de Salud de Porto Alegre (CAAE 34145314.5.3001.5338). El análisis de las entrevistas y de las observaciones se realizó con el soporte del software NVIVO. En una primera lectura del material empírico se realizó un análisis de contenido y, posteriormente, se identificaron los ejes centrales para la elaboración del análisis, con el apoyo del referencial teórico utilizado. Los ejes centrales de análisis fueron: 1) la influencia del contexto sobre los procesos de trabajo a nivel local, 2) la dinámica organizacional del servicio y 3) las percepciones individuales de las y los profesionales de la salud.

\section{RESULTADOS Y DISCUSIÓN}

En primer lugar, se sintetizan las características del universo empírico en el cual se realizaron las observaciones etnográficas, y se mapean los elementos que pueden influenciar en la oferta de la 
PEP en el servicio estudiado. El servicio está situado en la región atravesada por problemas sociales con relación al acceso a la vivienda, el saneamiento básico y la creciente violencia urbana. Como muestra la bibliografía, tales factores se pueden manifestar en el proceso salud-enfer$\operatorname{medad}^{(28)}$, lo cual influye en la producción de inequidades y vulnerabilidades en las personas $-\mathrm{y}$, en cierta medida, en los procesos de trabajo- que afectan a las y los profesionales de la salud que actúan en el territorio estudiado.

Desde el punto de vista del ambiente organizacional, el servicio está conformado por el centro de testeo y consejería y el servicio ambulatorio, ambos creados en la década de 1990. En sus primeros años, eran autónomos y mantenían coordinaciones distintas. En 2012, se inició el proceso de unificación de los servicios y gerencias, para actuar de forma conjunta en la asistencia especializada en ITS/VIH/sida. A pesar de la unificación, ambos servicios se mantuvieron, en la práctica, como unidades distintas desde el punto de vista organizacional, tanto en los flujos de atención como en los equipos, aunque estuvieran dentro de un mismo edificio. Cuando se realizó la investigación de campo, la conexión entre las unidades se resumía a una coordinación común, responsable de alinear técnicamente las acciones asistenciales y de testeo, y realizar reuniones conjuntas entre los equipos del centro de testeo y consejería y el servicio ambulatorio. La composición de ambos equipos era distinta e independente entre sí: los procedimientos y las acciones cotidianas de las unidades eran específicos de cada unidad.

El centro de testeo y consejería era responsable de realizar las pruebas convencionales -realizadas exclusivamente con el test Elisa y las pruebas confirmatorias - la consejería pre y postest, además de acciones de acompañamiento de las personas que asistían a este servicio. La atención psicosocial en el centro de testeo y consejería tenía, en promedio, una duración de una hora (era más prolongada que la duración promedio de la consejería en el servicio ambulatorio para situaciones de PEP). La oferta de pruebas convencionales se daba por demanda libre $y$, antes o después de la consejería, se derivaba a las personas al laboratorio y los resultados demoraban, en promedio, 15 días hábiles. La entrega del resultado se realizaba en el marco del acompañamiento y apoyo psicosocial y posconsejería. Al momento de realizar la investigación, el centro de testeo y consejería no ofrecía el test rápido para todas las personas, y restringía su uso a los casos de demanda de PEP.

El servicio ambulatorio concentraba acciones de asistencia y se centraba en el tratamiento y acompañamiento de personas que viven con VIH. No solía ofrecer test rápido o pruebas convencionales por demanda espontánea. Quien buscaba hacerse un test de VIH y demás ITS en el servicio ambulatorio, sin vínculo con el servicio, era derivado a su unidad de atención primaria de referencia, y no al centro de testeo y consejería. Según los entrevistados, la derivación a la atención primaria seguía la directriz propuesta por la gestión municipal, basada en orientaciones del Ministerio de Salud ${ }^{(29)}$. La oferta de PEP era una atribución exclusiva del servicio ambulatorio, desde la implementación de esa estrategia en 2010 y no fue alterada con la unificación de las gerencias de los servicios.

\section{Las dinámicas de la implementación}

La atención de personas que pasaron por una situación de exposición sexual es un momento complejo. En el servicio de asistencia especializada en ITS/VIH/sida, se observó que el ingreso se realizaba cuando, en la recepción del servicio, la persona relataba una situación de exposición sexual. En ese momento, las y los profesionales de la recepción acordaban una consulta inicial de evaluación para el uso de profilaxis.

Solo dos profesionales de enfermería concentraban la función de atender las situaciones de exposición en relaciones sexuales consensuadas y eran responsables de evaluar el riesgo a través del test rápido de VIH y sífilis, de la consejería y la recomendación del uso de profilaxis. La recepción, la evaluación y la consejería era un proceso que duraba en el servicio ambulatorio unos veinte minutos. Si la situación de exposición era considerada de riesgo significativo de infección por VIH, se ofrecía el uso de la PEP. Esta indicación se acompañaba con información sobre los efectos adversos, las interacciones medicamentosas y con otras sustancias.

Incluso con la indicación de PEP, la decisión sobre su uso le correspondía al usuario. Si 
este optaba por recibir la PEP, el profesional de enfermería se dirigía al consultorio médico, con el objetivo de discutir el caso, obtener una prescripción de antirretrovirales y de exámenes de laboratorio para monitorear el uso de PEP. Durante el trabajo de campo, se observó que las situaciones de exposición sexual siempre eran evaluadas por el personal de enfermería y no se observó una interacción directa de los usuarios con algún miembro del equipo médico. En el momento en que el usuario enunciaba su decisión de aceptar la PEP y que la enfermería se dirigía hacia el consultorio médico, se aplicaba el test rápido para VIH y sífilis. Los resultados de estos test estaban listos en aproximadamente veinte minutos. Mientras aguardaba los resultados y el retorno del equipo de enfermería, el usuario le manifestaba al investigador un sentimiento de ansiedad y de culpa debido a la situación potencial de exposición sexual.

Cuando el profesional regresaba al consultorio, se le comunicaba al usuario el resultado de los test rápidos. En el caso de que el resultado diera positivo para VIH, se derivaba al usuario a la red de atención primaria para iniciar el tratamiento en el servicio de salud más próximo a su casa, lo que podría demorar días o hasta meses en Porto Alegre. En el caso de que el resultado del test rápido de VIH diera negativo, el usuario recibía orientación sobre el uso de los antirretrovirales y se le explicaba, además, sobre el soporte asistencial en caso de necesidad. Por último, el profesional agendaba la nueva consulta en de 28 días. Se observó que las y los profesionales de enfermería eran enfáticos sobre la importancia de que el usuario asistiera a esta consulta programada, principalmente para realizar un nuevo test rápido y descartar alguna posibilidad de infección por VIH.

En el caso del centro de testeo y consejería, aunque no ofrecía PEP, se observaron posibles situaciones elegibles para la profilaxis. El investigador detectó dos flujos por los cuales el usuario en situación de exposición sexual podía ser identificado en el centro de testeo y consejería. El primero, en la recepción y, el segundo, durante la atención psicosocial. De acuerdo con las y los profesionales de la recepción, cuando las personas relataban situaciones de exposición sexual, eran derivados en forma inmediata al servicio ambulatorio. El segundo flujo se identificó en el momento de la atención psicosocial realizada por los consejeros profesionales. La identificación de estos casos era difícil y sucedía solo cuando el usuario relataba al profesional una situación de exposición reciente. A pesar del procedimiento establecido por el centro de testeo y consejería, hubo más de una situación durante el trabajo de campo en la que se observó, en el marco de la consejería pre o postest, el relato de una exposición reciente de riesgo al VIH con indicación de PEP y que no fue derivada al servicio ambulatorio.

Las entrevistas con profesionales del centro de testeo y consejería revelaron que percibían la unidad como un servicio en sí mismo y no como parte de un servicio especializado, por lo que consideraban que su funcionamiento era independente respecto del servicio ambulatorio. Al mismo tiempo, no había un protocolo claro que organizara el flujo de atención del usuario entre ambos espacios. Ante esta situación, no había una iniciativa por parte del centro de testeo y consejería de buscar activamente a aquellas personas que podrían beneficiarse de la PEP. Otro punto a destacar, se refiere al instrumento utilizado por los consejeros para realizar la atención psicosocial. El instrumento era un cuestionario estandarizado, el cual buscaba relevar información personal del usuario y sobre la motivación para realizarse el test. El cuestionario indagaba sobre exposiciones sexuales de riesgo que no habían ocurrido en las últimas 72 horas, sino en los últimos 12 meses. Así, la desactualización del instrumento contribuía a no investigar las situaciones de exposición, perjudicando el acceso a la profilaxis.

\section{Incorporación y desconocimiento}

Las y los profesionales entrevistados recordaban vagamente la incorporación de la PEP a la rutina del centro de testeo y consejería y al servicio ambulatorio. Los pocos relatos sobre ese proceso provenían de personas que trabajaban en el servicio ambulatorio, quienes percibían la incorporación de la PEP como un proceso realizado "de arriba hacia abajo", como una estrategia que recaía en el universo local de los servicios, sin contar con acciones de capacitación o educación continua. $\mathrm{Al}$ aspecto, los entrevistados del servicio ambulatorio relataron que no recordaban que 
se hayan realizado acciones de capacitación sobre PEP cuando se implementó en 2010. Tampoco recordaban acciones de educación continua para actualizar sus conocimientos sobre métodos de prevención con base en el uso de medicamentos antirretrovirales. En el centro de testeo y consejería se verificaron relatos similares.

En el proceso de implementación de políticas públicas, Sabatier indica la existencia de dos modelos principales: el modelo top-down (de arriba hacia abajo) y el modelo bottom-up (de abajo hacia arriba $)^{(30)}$. El top-down está fundamentado en una visión tecnicista, funcionalista y gerencial de toma de decisiones en la esfera política, mientras el modelo bottom-up busca promover un proceso de autoorganización y de participación de los actores que lidian directamente con lo público, considerados fundamentales en la toma de decisiones. En este estudio, la percepción de los entrevistados caracteriza una implementación top-down de la $\mathrm{PEP}$, lo que dificultó la incorporación de la estrategia en el servicio estudiado.

Los problemas iniciales de implementación pueden haber influido en la decisión de restringir el conocimiento sobre $\mathrm{PEP}$ a unos pocos profesionales. El conocimiento técnico-normativo sobre PEP se concentraba en los enfermeros del servicio ambulatorio. La gerencia y los colegas de servicio no estimulaban a estos profesionales a difundir el conocimiento que detentaban de los protocolos de la PEP. La ausencia o poco conocimiento sobre el plano normativo de una acción, estrategia o programa es señalado en la bibliografía como perjudicial para el proceso de implementación de las políticas públicas(31).

Además de eso, la ausencia de acciones de capacitación o educación continua sobre PEP puede contribuir a la creación de nociones individuales sobre la estrategia, e influir en los procesos de trabajo y la finalidad de la medida preventiva. Aunque las y los profesionales entrevistados reconocían la importancia de la PEP, eran reticentes respecto de los cambios que pueden generar las nuevas estrategias (sobre todo PEP y PrEP) en las políticas públicas de VIH/sida, lo cual puede representar una barrera para la implementación de la estrategia.

\section{El sujeto reincidente}

La importancia de la estrategia de la PEP fue reconocida por la mayoría de los entrevistados, aunque el uso del método quedara restringido a una forma de corregir situaciones que son indeseadas o, idealmente, no esperadas. Esta concepción distancia el uso del método como una estrategia preventiva, para ser incorporada en la práctica de los individuos de acuerdo con sus necesidades. Además, las y los profesionales cuestionaban la efectividad y el grado de protección otorgado por el método, y alertaban sobre la posibilidad de falla de la PEP. Así, la PEP es vista como una medida excepcional, para situaciones en las que falla la prevención del VIH:

[La PEP] es válida, porque los accidentes suceden realmente. Pero, a veces, ves que hay personas que reinciden. Vienen más de una vez. Ah, vienen dos, tres, cuatro veces. Entonces, ves que estas personas, incluso conociendo el riesgo, corren el riesgo ¿sabés? $Y$ va a llegar un momento que puede ser que no funcione. Comienzan a tomar la medicación, a tomar la medicación y ¿qué tal si alguna vez no funciona...? Se arriesgan bastante, pero creo que es muy válido tener esa garantía. (Entrevista, agosto de 2015)

En este relato, es posible percibir la preocupación por el uso reiterado de la estrategia. Las y los profesionales, en general, temían que el usuario elija tener sexo sin protección, incluso ante posibles riesgos de infección por VIH. Dicho de otro modo, se temía la banalización de la estrategia. Vale nuevamente destacar que la bibliografía no muestra una relación entre el uso de la PEP y el abandono de otros métodos de prevención del VIH(32).

El uso reiterado apareció en diversos relatos como un no-cuidado de la salud sexual por parte del usuario, y como un indicador del abandono de estrategias tradicionales de prevención del VIH (como el uso de preservativos y de gel lubrificante). Es verdad que la misma situación podría ser leída por las y los profesionales como una forma de gestión del riesgo $y$, en este sentido, una forma de cuidar la propia salud sexual (aunque no sea la forma de cuidado idealizada o propuesta 
por ellos). En este estudio, no se realizó un relevamiento de cuántos usuarios efectivamente hacían uso reiterado de la PEP; lo que subrayamos es que parte de las y los profesionales que trabajaban en el servicio ambulatorio tenían una concepción arraigada de que había usuarios que regresaban con frecuencia al servicio. No se constató tal concepción entre las y los profesionales del centro de testeo y consejería por el hecho de que estos no atendían, en la práctica, a las personas de PEP.

En la siguiente situación, el entrevistado relata cómo la pareja de una persona que vive con VIH hace uso reiterado de la estrategia:

Hay algunas [...] situaciones de repetición. Hay una chica que creo que vino acá, tal vez unas seis, siete veces. Así, prácticamente un mes sí, un mes no. Ella estaba casada y su marido tenía VIH y, en su situación, se le rompía el preservativo. La primera vez, la orientamos y todo: "mirá, él tiene una carga viral indetectable, vos vas a usar el medicamento" [...]. Al poco tiempo, de nuevo. "No sabés usar el preservativo, ¿lo estás lubrificando bien?”. Ahí le explicamos... Tercera vez, de nuevo. ¡Algo anda mal! ¡Ella sería una candidata a la PrEP! (Entrevista, agosto de 2015)

Cabe señalar que la oferta de la PrEP, al momento de realizar el trabajo de campo, aún no se encontraba disponible en el SUS. En el relato anterior, también llama la atención la indicación de uso de la PEP por el hecho de tener una relación sexual con una pareja que vive con VIH y carga viral indetectable. El riesgo estimado de transmisión en esos casos es insignificante e insuficiente para la indicación de PEP o PrEP. Ante esta situación, ONUSIDA lanzó en 2017 la campaña "Indetectable = Intransmisible", con el propósito de informar a gestores, profesionales y a la comunidad en general sobre la no transmisibilidad del VIH cuando la carga viral es indetectable, y sobre la importancia del tratamiento como forma de prevención del VIH(33).

Además, según el relato anterior, la rotura del preservativo es percibida como una indicación de mal uso del insumo o incluso de menor cuidado por parte del usuario. Es posible percibir que, en algunos momentos, las y los profesionales cuestionaban la veracidad de los relatos de rotura del preservativo. Esta situación puede darse por diferentes motivos como, por ejemplo, calidad inadecuada del insumo o un uso no adecuado. Por lo tanto, no debería resultar extraño que el motivo de demanda de PEP sea por la falla del método de prevención.

Las percepciones de uso reiterado de la PEP y la idea de abandono o del mal uso del preservativo inscriben significados sobre los sujetos y sus prácticas sexuales. Es el caso de la noción de sujeto reincidente, categoría emic identificada en el discurso de entrevistados:

Lo que la gente ve, creo que es una cosa que tal vez dé para comentar, es que hay personas que vienen varias veces. El sujeto que es reincidente. Eso lo vemos mucho: "ifulano!, ¿vos de nuevo por acá?". Lo ves... Porque normalmente, hoy en día, pasa lo siguiente: a vos te da pánico ir al cabaret e incluso sin exponerte, venís acá sudando e infartado... Ese tipo de actividad no es para vos, ¿no? (Entrevista, agosto de 2015)

Entre los entrevistados, el sujeto reincidente puede asumir dos facetas: en una, es aquel sujeto irresponsable, pues conscientemente incurre reiteradas veces en situaciones de potencial exposición sexual al VIH, sobrecargando el servicio cada vez que retorna para acceder a la PEP. La idea de que este sujeto asume un riesgo es lo que hace que sea visto como irresponsable por los entrevistados, a pesar de haber ido al servicio en busca de un cuidado y de un método preventivo, que no puede comprar en una farmacia u obtener en un servicio particular. Otra faceta del sujeto reincidente es aquella que se preocupa excesivamente: tanto con relación al riesgo de infección por VIH, como por el estigma asociado al VIH y al sida. A este sujeto preocupado, en algunos casos, las y los profesionales de la salud lo evalúan como no-calificado para el uso del método, dado que ni siquiera consigue asumir socialmente la práctica que tuvo, lo cual expresa otra punición al individuo.

\section{La no-urgencia de la prevención}

La PEP es una urgencia médica, según el protocolo $^{(1)}$. En Porto Alegre, la PEP está disponible en 
los servicios de asistencia especializada en ITS/ $\mathrm{VIH} /$ sida y en los servicios de emergencia y urgencia. La implementación de la PEP en esos servicios no fue objeto de este estudio; sin embargo, nos interesa sumar a la discusión el modo en que las y los profesionales del servicio de asistencia especializada en ITS/VIH/sida perciben la oferta de la PEP en los servicios de emergencia/urgencia médica en Porto Alegre. Los entrevistados percibían que, fuera del servicio, la PEP no solía ser entendida ni como una emergencia ni como una urgencia médica; no diferenciaban la emergencia de la urgencia, pero resaltaban que la PEP en las situaciones de sexo consensuado no era vista como un problema que necesitaba de una intervención médica inmediata. En algunas consultas clínicas observadas en el servicio ambulatorio, se verificaron situaciones en las que las personas provenían de servicios de emergencia/urgencia, en los cuales intentaron acceder al uso de profilaxis, sin éxito.

Por otro lado, las situaciones que involucraban exposición por violencia sexual, según la percepción de los entrevistados, eran encaradas de forma diferente por los mismos servicios de emergencia/ urgencia. Al respecto:

Entonces, comenzaron con las emergencias también. Ahí las emergencias comenzaron así: es violencia, hay que atenderlo; no es [violencia], no se atiende, dejalo, que venga otro día. Se dio tal cual así: fue estupro, fue esto, aquello, se atiende; si no, entonces "vas a venir otro día, porque no es necesario atenderte". Porque tipo así: "ah, lo hiciste, ahora arreglate" (Entrevista, agosto de 2015)

No es solo entre profesionales de la salud de otros servicios de salud que la PEP era vista de esta forma. La percepción de no-urgencia también era compartida por algunos entrevistados, los cuales entendían como dificultosa la atención de los casos de exposición sexual con mayor riesgo al VIH en servicios de emergencia/urgencia:

Entonces, a veces, creo que está mal atender PEP en la guardia... Ya trabajé en la guardia: estás con un tipo con un infarto, $y$ con otro que está con una paresia, no sabés si el tipo va hacia un coma o no, i¿y alguien resolvió tener sexo y no usar preservativo en el medio de tu guardia?! Es complicado, ¿no? Ahí tenés que parar todo cuando tenía que estar al lado del otro [paciente infartado, etc.]. En la guardia del SUS... siempre falta gente, entonces estás vos para todo. Y... las cosas caen en tus manos, entonces yo... yo lo veo mal, porque el tipo termina haciendo cualquier cosa para librarse del paciente [PEP]. (Entrevista, agosto de 2015)

El relato anterior revela que la potencial exposición al VIH, producto de una relación sexual consensuada, frente a otros casos graves de salud, es interpretada como un evento que no exigiría una atención médica inmediata. Además, es posible identificar cómo la percepción de carencia de recursos humanos en los servicios de emergencia/ urgencia también influye en la noción de que la exposición sexual consensuado no necesita de atención inmediata.

A la percepción de no-urgencia médica se suma la idea de culpabilización del usuario. Las y los profesionales percibían que, en los servicios de emergencia o urgencia, el no-uso del preservativo o de cualquier otro medio de prevención del VIH se lo consideraba un acto voluntario del usuario que eligió ser irresponsable. Ya en el caso de violencia sexual, por el hecho de no mediar una elección, no se responsabilizaba a la víctima, la cual tenía atención prioritaria. La posibilidad de evitar la transmisión del VIH luego de una relación sexual -consensuada o no- era reconocida por las y los profesionales. Sin embargo, no parecía haber consenso entre los entrevistados respecto de la atención prioritaria en los servicios de emergencia/urgencia para las situaciones de exposición sexual consensuada ante otros problemas de salud, lo cual difiere de lo propuesto en el protocolo de PEP.

\section{Desafíos para la implementación}

La actuación de las y los profesionales permite corroborar las lagunas en el diseño y la ejecución de las estrategias de salud. En esta sección, se identifican los desafíos mencionados por las y los profesionales para la implementación de la PEP. Se observó una mayor divergencia en las 
concepciones entre las y los profesionales del servicio ambulatorio y del centro de testeo y consejería, que diferencias en las percepciones y el conocimiento sobre la profilaxis entre la gerencia del servicio y los demás entrevistados.

Se constató la necesidad de mejorar la coordinación organizacional entre el servicio ambulatorio y el centro de testeo y consejería en la atención de los casos de exposición sexual consensuada. La concentración de la PEP en el servicio ambulatorio dificulta la realización de acciones específicas de apoyo psicosocial, que se ofrecían sobre todo en el centro de testeo y consejería. Más allá de que el servicio ambulatorio realizara acciones de consejería, según un entrevistado, había una necesidad de mayor atención a esa actividad:

No, creo que, de desafíos, así, tendríamos que mejorar mucho, creo que debería haber un profesional disponible para atender este tipo de situaciones, de aconsejar, de tener más tiempo de hacer una buena consejería. (Entrevista, agosto de 2015)

En el relato anterior también se verifica la necesidad de un profesional de consejería disponible exclusivamente para PEP. Esta percepción del entrevistado puede ser interpretada como un efecto de la concentración de actividades relativas a la PEP en pocos profesionales. De esa forma, en vez de que el equipo se apropie de la consejería, como tarea importante en la atención de PEP, se la percibe como atribución de unos pocos profesionales.

Según un entrevistado, en vistas de un posible aumento de la demanda por PEP, se verificó la necesidad de incrementar las acciones de gestión pública en los diferentes niveles (municipal, estatal y federal) dirigidas a la planificación de las actividades relacionadas con la prevención antirretroviral:

Entonces, creo que los gestores deberían ya estar presentes, viendo el aumento de la demanda y estar previendo la capacitación de la red para absorber esa demanda, preparación, capacitación, de hacer una proyección, esto tiene que ver con la gestión. Porque no es una cosa loca, una cosa del servicio, eso es una cosa que viene del Ministerio [de Salud], una cosa nacional y que tiene... que está prevista. Creo que no está de esa forma todavía, por lo menos no escuché nada en el sentido de "bueno, vamos a preparar la atención primaria”. Creo que eso está muy incipiente. (Entrevista, agosto de 2015)

El desafío, en este caso, es la ampliación de la oferta de PEP en los servicios públicos de salud. Según otro entrevistado, la oferta se limitaba a pocos servicios, loba que obliga al usuario a desplazarse por diferentes zonas del municipio en busca de la profilaxis:

Creo que debería haber más lugares disponibles [para la atención de $\mathrm{PEP}$ ], porque a veces es difícil. La gente del centro, allá de la zona norte, tiene que desplazarse hasta acá ¿no? A veces, ese desplazamiento de la persona... Viene el que puede y quien lo busca pero, a veces, en un mal horario, porque es de 8 a 17 [funcionamiento del ambulatorio], $y$ las personas trabajan. Y va a salir del trabajo y va a decir: "¿voy ahí a hacerme un test rápido?" Las personas no se van a exponer, ¿no? (Entrevista, agosto de 2015)

Se observa la dificultad de acceder a la PEP en los servicios especializados, por el horario de funcionamiento de estos servicios. Sobre este punto, se destaca que, independientemente de la oferta de PEP en los servicios de emergencia/urgencia, abiertos las 24 horas, en la percepción de los entrevistados estos servicios no son de fácil acceso en los casos de exposición sexual consensuada. Cuando el entrevistado afirmaba que las personas no realizarían la profilaxis en horario comercial debido a la posibilidad de exposición pública, se observa que el estigma, de modo general, persiste como una barrera en el acceso a la estrategia. Tal afirmación se suma a las demás percepciones negativas sobre las personas en busca de prevención antirretroviral.

Se observó, también, la cuestión de la difusión de información sobre la PEP:

Las personas no se están previniendo, no lo están usando. Entonces, intentamos hacer, a través de los organismos públicos, alguna cosa más... Claro que se está intentando hacer 
divulgación, pero si solo se hace divulgación, "ah, se usa medicación solo si sucede esto". Pero ¿y la prevención para no llegar a usar el medicamento? ¿Cómo están usando la cuestión para prevenir? ¿Para usar el preservativo? No necesita llegar a ese punto [...] Que la cosa se divulgue más, o hacer el trabajo, no sé, de salir a la noche, o lo que la gente... como se hacía antes con la reducción de daños, ¿no? Algo hay que hacer, porque así jes demasiado! (Entrevista, agosto de 2015).

La percepción de la necesidad de divulgación de la PEP es recurrente y paradójica: al mismo tiempo que los entrevistados discutían la necesidad de una mayor divulgación de la estrategia en diferentes espacios, revelaban el temor de una posible banalización del sexo desprotegido, promovido por las nuevas tecnologías. El énfasis entonces se ponía en la divulgación de estrategias de prevención en general, retomando, por ejemplo, la lógica de la reducción de daños y, especialmente, la distribución de preservativos y del gel lubricante. Se retornaba a la cuestión de que el fenómeno del abandono del uso de métodos tradicionales por parte de la población ${ }^{(16)}$ aún no se había incorporado en las reflexiones de estos profesionales, ni lo habían considerado como un fenómeno que puede ser independente de las innovaciones aportadas por la prevención combinada.

\section{Nuevos dilemas de las políticas de prevención}

La prevención basada en el medicamentos antirretrovirales, traducida sobre todo por las tecnologías PEP y PrEP, ha provocado transformaciones en las políticas públicas de prevención del $\mathrm{VIH}^{(2)}$, que se ven reflejadas en ciertas inconsistencias en la forma en la que están siendo implementadas en los servicios públicos de salud.

Desde inicios de la década de 1990, los servicios estudiados están formados por profesionales que participan del proceso de incorporación de diferentes estrategias en el campo de la prevención, desde intervenciones biomédicas hasta intervenciones comportamentales y estructurales. Para algunos entrevistados, el actual escenario se enfoca demasiado en las intervenciones basadas en el uso de antirretrovirales, especialmente PEP y PrEP y, aunque reconocen la importancia de estos métodos, señalan la reducción de estrategias con enfoque comportamental y socioestructural.

Verificamos la preocupación de los entrevistados por cuestiones socioestructurales que dificultan la prevención del VIH. La bibliografía señala que enfrentar al VIH exige la asociación de estrategias (biomédicas, comportamentales y socioestructurales), relacionadas con las realidades de los sujetos $^{(3,4)}$. A las cuestiones señaladas, se suma la percepción de un drástico cambio en la estructura de los servicios, con la reducción de recursos humanos, cierre de centros de testeo, el agravamiento de cuestiones sociales como la violencia urbana y la desigualdad socioeconómica, las cuales, de forma directa o indirecta, influyen sobre los procesos de trabajo en el cotidiano de los servicios.

Las percepciones sobre la PEP aquí descritas transitan el conocimiento técnico-normativo, las subjetividades $y$, también, un contexto mayor que abarca la implementación de las acciones de las y los profesionales de la salud. Mientras la prevención antirretroviral se torna una realidad, el desfinanciamiento de la salud pública y la desestructuración de estrategias tradicionales, así como el contexto local, entre otros factores, generan desconfianza e inseguridad en los trabajadores. De allí que las percepciones no reflejan la mirada aislada de los entrevistados, sino una serie de eventos interrelacionados y que inciden en el ámbito local de los servicios de salud.

Otra cuestión que merece ser subrayada es la noción, antes mencionada, de sujeto reincidente, una lectura particular de las y los profesionales amparada en una connotación negativa sobre los sujetos y sus prácticas y la forma en que se apropian de la PEP. Eso revela la importancia de pautar acciones de promoción de equidad, no discriminación y respeto a las vulnerabilidades en los servicios ${ }^{(34)}$. Bajo tal percepción, el sexo protegido por la PEP es negado y pensado como una irresponsabilidad o negligencia. En ese sentido, la estrategia PEP, desde la percepción de las y los profesionales, se torna un elemento que denuncia la ausencia de autocuidado, para la cual el sujeto reincidente merece una reprimenda, pues la estrategia no es pensada por las y los profesionales como una forma de prevención. 
La reincidencia, categorización jurídica para aquel que incurre seguidamente en un crimen, es apropiada para expresar una idea de desvío o delito en la exposición sexual consensuada al VIH. De esa forma, le imprime una carga de moralidad prescriptiva al propio ejercicio de la sexualidad del sujeto. En este sentido, las expectativas relacionadas con la subjetivación, la responsabilización y el control de sí han sido atravesadas por nuevas tecnologías de poder ${ }^{(35)}$. La no utilización de métodos tradicionales rompe con la máxima de que el preservativo, sea el masculino o el femenino, son las "mejores" formas de prevención del VIH, idea que aún está vigente en campañas de prevención y en muchos discursos biomédicos. Aquí, tal vez el "mejor" mecanismo de prevención no esté definido por el que más se adapte al contexto de los sujetos, o al que cada uno evalúe como mejor para sí, en su vida, en ese momento.

La epidemia de VIH/sida es una epidemia de significaciones $^{(21)}$, en la cual el uso del lenguaje no es neutro, y refleja intereses y relaciones de poder. En el ámbito de los servicios de salud, la producción de significados sobre los sujetos y sus prácticas no son necesariamente reflejo de la realidad objetiva. La concepción negativa de reincidencia sobre el comportamiento sexual del sujeto puede ser atribuida al estigma sobre las prácticas sexuales consideradas desviantes y sobre el sida; estigma comprendido aquí como fenómeno que incide sobre los sujetos que, en virtud de su diferencia, son negativamente valorados por la sociedad $^{(36)}$. El sujeto que busca el servicio para acceder a la PEP puede ser interpretado como aquel con prácticas sexuales "promiscuas" (con muchas parejas), o "inmorales" (asociadas al trabajo sexual o a formas no tradicionales de relaciones sexuales y afectivos), por lo tanto, un sujeto cuya reincidencia lo torna un sujeto estigmatizado.

Como ya señalamos, es posible pensar al mismo sujeto reincidente bajo otra perspectiva: la del sujeto preocupado, en la que se puede pensar a este sujeto como aquel que invirtió tiempo y recursos buscando conocer la estrategia de la PEP y que se apropió de los medios para acceder a través de las políticas públicas de salud. Aquí, la reincidencia podría adquirir una connotación positiva: sería una práctica que indica una preocupación por la propia salud sexual, de alguien que adquirió el conocimiento necesario para tener autonomía sobre el uso de la estrategia y que, aunque encuentre restricciones en el acceso, busca acceder en el ámbito de los servicios públicos de salud. La prevención combinada presupone justamente un sujeto autónomo que puede decidir incluso sobre el uso de la PEP diversas y reiteradas veces. Es importante destacar que esta interpretación alternativa del sujeto reincidente, con connotación positiva, no fue verificada en la investigación.

El sujeto reincidente podría incluso ser pensado como una oportunidad para el servicio de acceder a un usuario con alto riesgo de infección por VIH y crear un vínculo con él. Este usuario de la estrategia de $\mathrm{PEP}$, reincidente o no, cuando llega al servicio ambulatorio, accede a la realización de tests rápidos (VIH, sífilis y hepatitis), la consejería pre y postest $\mathrm{y}$, eventualmente, tiene cubiertas otras demandas de salud, como la vacunación para la hepatitis y el VPH. En este caso, el usuario podría ser recibido con la idea de que vino al lugar correcto y siempre que lo necesite puede volver, para ser atendido cuantas veces quisiera o sea necesario. En la observación de cuño etnográfico, sin embargo, se percibió que la recepción tiene más un tono de reprimenda, en el sentido de "que no se repita más".

Las estrategias PEP y PrEP han sido priorizadas por el Ministerio de Salud para grupos poblacionales denominados como poblaciones-claves o poblaciones prioritarias: hombres que tienen sexo con hombres, gays, profesionales del sexo, travestis y transexuales. Se trata de una perspectiva de gestión pública que apunta a priorizar estas nuevas tecnologías de prevención del VIH para grupos considerados de mayor vulnerabilidad a la infección. Por lo tanto, el protocolo de prevención combinada incorpora, de alguna manera, la idea de vulnerabilidad. Sin embargo, el protocolo es solo uno de los elementos en la gestión pública, porque si no estuviera acompañado de otras estrategias que apunten a la promoción de derechos humanos -especialmente derechos sexuales y derecho a la no-discriminación- el protocolo por sí solo es poco eficaz en la ampliación del acceso a las nuevas tecnologías de prevención del

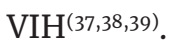

La reincidencia, de la misma forma que la culpabilización, crea un sujeto en el discurso y en la representación de las y los profesionales que actúa en la implementación de la profilaxis en el servicio 
observado. Los actos performativos, en la concepción de Butler(37), son también actos de lenguaje que no solo describen, sino que constituyen a los sujetos dentro de campos discursivos de poder y saber. Se observa que la sexualidad, en las situaciones de exposición en relaciones sexuales consensuadas es performada, al producir significados en las prácticas y en los cuerpos de los sujetos. El sujeto reincidente carga los marcadores sociales de clase, de género, de orientación sexual y de raza: es el usuario irresponsable, pobre, gay, promiscuo y no blanco. Los mismos marcadores que colocan a este sujeto como prioritario para el protocolo de la PEP funcionan como barreras para su acceso a la profilaxis. Tales prácticas inscriben estos cuerpos como ilegítimos para el uso de la PEP.

\section{CONSIDERACIONES FINALES}

El presente trabajo buscó identificar los desafíos de la implementación de la PEP, a partir del análisis de la actuación de profesionales de la salud, en un servicio público especializado en ITS/VIH/sida en Porto Alegre, Rio grande do Sul, Brasil. Entre los principales resultados se detectó la influencia de elementos contextuales, organizacionales y subjetivos, los cuales conforman y performan las acciones de implementación de la PEP en el servicio estudiado. Se analizó cómo tales elementos generan dificultades en el acceso a la PEP, desafiando la efectividad de la estrategia en una perspectiva de respeto a las vulnerabilidades y los derechos humanos de los sujetos.
La implementación de una política pública no es un proceso neutro, justamente porque está realizado por profesionales que interpretan las normas y los protocolos, y construyen consensos sobre la estrategia a nivel local del servicio. En ese sentido, las y los profesionales de salud, principales responsables de la traducción de las políticas públicas a los beneficiarios del SUS, están ante una paradoja: al mismo tiempo que el uso de antirretrovirales en la prevención del VIH -especialmente la PEP y la PrEP- se propaga internacional y nacionalmente como potencial mecanismo de enfrentamiento a la epidemia de VIH/sida, su materialización en la realidad local, a través de las percepciones analizadas, encuentra ruidos y potenciales barreras para el acceso a esta estrategia.

La idea de un sujeto reincidente en la PEP como preconcepción negativa sobre eventuales prácticas, se puede manifestar como discriminación en la recepción o durante la atención; e incluso puede agravarse con relación a grupos sociales específicos, por marcadores de clase, raza o color, expresión de género, sexualidad, discapacidades, entre otros. Por lo que se sugiere prestar mayor atención al proceso de implementación de métodos de prevención basados en el uso de medicamentos antirretrovirales en el escenario brasileño. Esto atraviesa el incremento de acciones dirigidas a la capacitación y a la educación continua de profesionales, tanto en relación con los aspectos técnico-normativos de protocolos, como en la promoción de equidad, no discriminación y respeto a los derechos humanos de los sujetos en el ámbito de los servicios de salud. 


\section{REFERENCIAS BIBLIOGRÁFICAS}

1. Ministério da Saúde. Protocolo clínico e diretrizes terapêuticas para profilaxia pós-exposição (PEP) de risco à infecção pelo HIV, IST e hepatites virais [Internet]. 2018 [citado 1 ago 2019]. Disponible en: https://tinyurl. com/toqekkp.

2. UNAIDS. Combination HIV prevention: tailoring and coordinating biomedical, behavioural and structural strategies to reduce new HIV infections. [Internet]. 2010 [citado 2 dic 2019]. Disponible en: https://tinyurl.com/ sy8c3xa.

3. Kippax S, Stephenson N. Beyond the distinction between biomedical and social dimensions of HIV prevention through the lens of a social public health. American Journal of Public Health. 2012;102(5):789-799.

4. Chang LW, Serwadda D, Quinn TC, Wawer MJ, Gray $\mathrm{RH}$, Reynolds SJ. Combination implementation for HIV prevention: moving from clinical trial evidence to population-level effects. The Lancet Infectious Diseases. 2013;13(1):65-76.

5. Grangeiro A, Kuchenbecker R, Veras MA. New HIV prevention methods: recognizing boundaries between individual autonomy and public policies. Revista Brasileira de Epidemiologia. 2015;18(Suppl 1):1-4.

6. Kenworthy N, Thomann M, Parker R. From a global crisis to the "end of AIDS": New epidemics of signification. Global Public Health. 2018;13(8):960-971.

7. Maksud I, Fernandes NM, Filgueiras SL. Tecnologias de prevenção do HIV e desafios para os serviços de saúde. Revista Brasileira de Epidemiologia. 2015;18:104-119.

8. Zucchi EM, Grangeiro A, Ferraz D, Pinheiro TF, Alencar T, Ferguson L, et al. Da evidência à ação: desafios do Sistema Único de Saúde para ofertar a profilaxia pré-exposição sexual (PrEP) ao HIV às pessoas em maior vulnerabilidade. Cadernos de Saúde Pública [Internet]. 2018 [citado 5 dic 2019];34(7):e00206617.

9. Grangeiro A, Ferraz D, Calazans G, Zucchi EM, DíazBermúdez XP. The effect of prevention methods on reducing sexual risk for HIV and their potential impact on a large-scale: a literature review. Revista Brasileira de Epidemiologia. 2015;18:43-62.

10. Poynten IM, Smith DE, Cooper DA, Kaldor JM, Grulich $\mathrm{AE}$. The public health impact of widespread availability of nonoccupational postexposure prophylaxis against HIV. HIV Medicine. 2007;8(6):374-381.

11. Poynten IM, Jin F, Mao L, Prestage GP, Kippax SC, Kaldor JM, et al. Nonoccupational postexposure prophylaxis, subsequent risk behaviour and HIV incidence in a cohort of Australian homosexual men. AIDS. 2009;23(9):1119-1126.
12. Jain S, Oldenburg CE, Mimiaga MJ, Mayer KH. Longitudinal trends in HIV non-occupational post-exposure prophylaxis (NPEP) use at a Boston community health center between 1997 and 2013. Journal of Acquire Immune Deficiency Syndromes. 2015;68(1):97-101.

13. Ferraz DAS. Experiências de mulheres usuárias de profilaxia pós-exposição sexual ao HIV (PEP sexual): cenários pessoais e programáticos para a prevenção da aids [Internet]. Universidade de São Paulo; 2018 [citado 17 nov 2019]. Disponible en: https://tinyurl.com/ qwuyvv7.

14. Ferraz D, Paiva V. Sexo, direitos humanos e AIDS: uma análise das novas tecnologias de prevenção do HIV no contexto brasileiro. Revista Brasileira de Epidemiologia. 2015;18:89-103.

15. Dourado I, MacCarthy S, Reddy M, Calazans G, Gruskin S, Dourado I, et al. Revisitando o uso do preservativo no Brasil. Revista Brasileira de Epidemiologia. 2015;18:63-88.

16. Ministério da Saúde. Pesquisa de conhecimentos, atitudes e práticas na população brasileira - PCAP 2013 [Internet]. Ministério da Saúde; 2016 [citado 26 jun 2019]. Disponible en: https://tinyurl.com/wf4who2.

17. Körner H, Hendry O, Kippax S. Safe sex after postexposure prophylaxis for HIV: intentions, challenges and ambivalences in narratives of gay men. AIDS Care. 2006;18(8):879-887.

18. Schechter M, do Lago RF, Mendelsohn AB, Moreira RI, Moulton LH, Harrison LH, et al. Behavioral impact, acceptability, and HIV incidence among homosexual men with access to postexposure chemoprophylaxis for HIV. Journal of Acquire Immune Deficiency Syndromes. 2004;35(5):519-525.

19. Lipsky M. Street-level bureaucracy: dilemmas of the individual in public service, 30th anniversary expanded edition. New York: Russell Sage Foundation; 2010.

20. Lotta G. O papel das burocracias do nível de rua na implementação de políticas públicas: entre o controle e a discricionariedade. En: Faria CAP, (org.). Implementação de políticas públicas: teoria e prática. Belo Horizonte: PUC Minas; 2012. p. 20-49.

21. Hill M, Hupe PL. Implementing public policy: An introduction to the study of operational govern. Los Angeles: Sage Publications; 2009.

22. May PJ, Winter SC. Politicians, managers, and streetlevel bureaucrats: Influences on policy implementation. Journal of Public Administration Research and Theory. 2009;19(3):453-476. doi: 10.1093/jopart/mum030.

23. Grangeiro A, Couto MT, Peres MF, Luiz O, Zucchi EM, de Castilho EA, et al. Pre-exposure and postexposure prophylaxes and the combination HIV prevention methods (The Combine! Study): protocol for a pragma- 
tic clinical trial at public healthcare clinics in Brazil. BMJ Open. 2015;5(8):e009021.

24. Grangeiro A. Projeto de pesquisa: A efetividade da profilaxia da transmissão do HIV pós-exposição sexual consensual (PEP sexual), do uso combinado dos métodos preventivos contra a infecção pelo HIV e da profilaxia pré-exposição sexual (PrEP). São Paulo: USP; 2015.

25. Ministério da Saúde. HIV AIDS 2018. Boletim epidemiológico HIV/Aids. 2018;49(53):1-66.

26. SUL 21. Porto Alegre, capital da Aids no país: "paradoxo em uma cidade moderna - ou que já foi moderna" [Internet]. 2019 [citado 18 nov 2019]. Disponible en: https://tinyurl.com/wa4v872.

27. Johnson JM, Rowlands T. The interpersonal dynamics of in-depth interviewing. En: The SAGE handbook of interview research: The complexity of the craft. 2nd ed. California: SAGE Publications; 2012. p. 99-114.

28. Barata RB. Como e por que as desigualdades dociais fazem mal à saúde. Rio de Janeiro: Fundação Oswaldo Cruz; 2009.

29. Ministério da Saúde. Guia orientador para a realização das capacitações para executores e multiplicadores em teste rápido para HIV e sífilis e aconselhamento em DST/Aids na atenção básica para gestantes. Brasília: Ministério da Saúde; 2013.

30. Sabatier PA. Top-down and bottom-up approaches to implementation research: a critical analysis and suggested synthesis. Journal of Public Policy. 1986;6(1):2148 .
31. Wilson JQ. Bureaucracy: what government agencies do and why they do it. New York: Basic Books; 1989.

32. Lunding S, Katzenstein T, Kronborg G, Lindberg J, Jensen J, Nielsen $\mathrm{H}$, et al. The Danish PEP registry: experience with the use of postexposure prophylaxis (PEP) following sexual exposure to HIV from 1998 to 2006. Sexually Transmitted Diseases. 2010;37(1):49-52.

33. UNAIDS. Indetectável=Intransmissível: Saúde Pública e supressão da carga viral do HIV [Internet]. 2018 [citado 19 jun 2019]. Disponible en: https://tinyurl.com/wlz96tb.

34. Paiva V, Pupo LR, Barboza R. O direito à prevenção e os desafios da redução da vulnerabilidade ao HIV no Brasil. Revista de Saúde Pública. 2006;40:109-119.

35. Carrara S, Carrara S. Moralidades, racionalidades e políticas sexuais no Brasil contemporâneo. Mana. 2015;21(2):323-345.

36. Goffman. Estigma: Notas sobre a manipulação da identidade deteriorada. 4ta ed. Rio de Janeiro: LTC; 1981.

37. Butler J. Bodies that matter: On the discursive limits of sex. Abingdon: Routledge; 2011.

38. Edmundo K, Mercadante R. Planejar para quem? Quem planeja? A quem priorizar? En: Paiva V, França Jr. I, Kalichman AO, (org.). Vulnerabilidade e direitos humanos Prevenção e promoção da saúde. Curitiba: Editora Juruá; 2012. p. 249-270.

39. Rios RR, Silva R. Democracia e direito da antidiscriminação: interseccionalidade e discriminação múltipla no direito brasileiro. Ciência e Cultura. 2017;69(1):44-49.

\section{FORMA DE CITAR}

Kauss B, Leal AF, Grangeiro A, Couto MT. Reincidentes en el cuidado, pero sin derecho a la prevención: un análisis de la oferta de la profilaxis posexposición sexual al VIH en Porto Alegre, Brasil. Salud Colectiva. 2020;16:e2463. doi: 10.18294/sc.2020.2463.

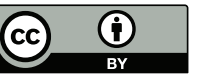

Esta obra está bajo una licencia Creative Commons Atribución 4.0 Internacional (CC BY 4.0). http://creativecommons.org/licenses/by-nc-nd/4.0

Atribución - Se debe dar crédito de manera adecuada, brindar un enlace a la licencia, e indicar si se han realizado cambios. Puede hacerlo en cualquier forma razonable, pero no de forma tal que sugiera que usted o su uso tienen el apoyo de la licenciante. Sin restricciones adicionales — No se pueden aplicar términos legales ni medidas tecnológicas que restrinjan legalmente a otras personas a hacer cualquier uso permitido por la licencia.

Recibido: 9 ago 2019 | Versión final: 10 dic 2019 | Aprobado: 06 mar 2020 | Publicado en línea: 27 mar 2020 